\title{
Positive Linear Maps on Operator Algebras
}

\author{
David E. Evans \\ School of Theoretical Physics, Dublin Institute for Advanced Studies, Dublin 4, Ireland
}

\begin{abstract}
Given a family of completely positive maps, indexed by a group, from a $C^{*}$-algebra into itself, we are concerned with its dilation to a group of *-automorphisms on a larger algebra. A Schwarz-type inequality for $n$-positive *-linear mappings from an involutive algebra into the bounded linear operators on a hilbert space is obtained. Strongly continuous oneparameter semigroups and groups on $C^{*}$-algebras, which have certain positivity properties, are studied.
\end{abstract}

\section{Introduction}

Given a family of completely positive maps, indexed by a group $G$, from a $C^{*}$ algebra into itself, we are concerned with its dilation to a group of *-automorphisms on a larger algebra. Theorem 1 is an operator algebra analogue of B.Sz.Nagy's hilbert space [12], and E.Stroescu's Banach space [11] dilations. This generalises to an arbitrary group some of the work of E. B. Davies [4] within the theory of quantum stochastic processes, and that of G.Lindblad [9] for quantum dynamical semigroups (both working in the Schroedinger picture).

We obtain a Schwarz-type inequality for certain $n$-positive mappings on involutive algebras. This produces some results for strongly continuous oneparameter semigroups and groups on $C^{*}$-algebras, which have various positivity preserving properties, e.g. 2-positivity, complete positivity. This extends the work of G.Lindblad [9] on the infinitesimal generators of norm continuous semigroups of completely positive maps. The general problem of characterising the infinitesimal generators of strongly continuous semigroups of completely positive maps is very difficult. As in the hilbert space situation, we expect that progress will be made in the other direction in obtaining techniques for constructing generators using more and more complicated unbounded dissipative and hamiltonian terms.

We remark that the physical significance of completely positive maps as operations on quantum systems has been observed in $[8,9]$. 
This work was carried out at The Mathematical Institute, Oxford. The author would like to thank E. B. Davies for all his help and encouragement, and the Science Research Council for their financial support during this period.

\section{Dilations of Completely Positive Maps on Operator Algebras}

If $A$ is a *-algebra, and $B$ is a $C^{*}$-algebra, let $w$ be a linear map from $A$ into $B$. Then if $n \in \mathbb{N}$, we say $w$ is $n$-positive if for all $\left\{x_{i} ; i=1,2, \ldots, n\right\}$ in $A$,

$$
\left[w\left(x_{i}^{*} x_{j}\right)\right] \geqq 0 \text { in } B_{n},
$$

where $B_{n}$ denotes the $C^{*}$-algebra of all $n \times n$ matrices over $B . w$ is said to be completely positive (CP) if $w$ is $n$-positive for all $n \in \mathbb{N}$. Suppose $A$ is a Banach *-algebra with approximate identity, and $B=B(\mathscr{H})$, all the bounded linear operators on a Hilbert space $\mathscr{H}$. Then it is known $[3,6,10]$ that a bounded linear map $w: A \rightarrow B(\mathscr{H})$ is completely positive if and only if there exists another Hilbert space $R$, a bounded linear map $V$ from $\mathscr{H}$ into $R$, and $a^{*}$-representation $\pi$ of $A$ on $R$ such that

$$
w(x)=V^{*} \pi(x) V \quad \forall x \in A,
$$

in which case, $(\pi, v)$ is said to be minimal [3] if $[\pi(A) V \mathscr{H}]=R$. A minimal pair for $w$ is uniquely determined up to unitary equivalence, and written $\left(\pi_{w}, V_{w}\right)$.

The following theorem can be regarded as the $C^{*}$-algebra analogue of E.Stroescu's Banach space dilations [11, Th. 1]. For technical reasons, we will omit from our treatment continuity considerations when $G$ is a topological group.

Theorem 1. Let $A$ be a $C^{*}$-algebra with identity and $G$ a group. For each $g$ in $G$, $w_{g}$ is a CP map of $A$ into itself such that $w_{g} 1=1$ and $w_{1}=1$. Then there exists $a$ $C^{*}$-algebra $B$ containing $A$, and a homomorphism $\Phi$ from $G$ into the *-automorphism of $B$, and a normal conditional expectation $N$ from $B$ onto $A$ satisfying:

i) $N\left(\Phi_{g} a\right)=w_{g}(a) \quad \forall g \in G, a \in A$.

ii) $B$ is the $C^{*}$-algebra generated by $\left\{\Phi_{g} a: g \in G, a \in A\right\}$.

iii) The identity of $A$ is also an identity for $B$.

Proof. Take $A$ to be faithfully represented as a concrete $C^{*}$-algebra on a Hilbert space $\mathscr{H}$. For each $g$ in $G$, let $\pi_{g}, V_{g}, R_{g}$ denote the Stinespring representation $\pi_{w_{g}}$, etc. Then $R_{1}=\mathscr{H}, V_{1}=1, \pi_{1}=1$, and $V_{g}$ is an isometry for all $g$ in $G$. Define $R=\bigoplus_{g \in G} R_{g}$, and $B_{g}=B(R)$ for all $g$ in $G$, and put $B_{0}=\bigoplus_{g \in G} B_{g}$. For each $g$ in $G$, we will define a unitary operator $U_{g}$ on $R$.

Put $U_{1}=1$, and for $g \in G, g \neq 1$,

$$
\text { define }\left(U_{g} \xi\right)_{h}= \begin{cases}\xi_{h} & h \neq 1, g \\ V_{g} \xi_{1}+\left(1-V_{g} V_{g}^{*}\right) \xi_{g} & h=g \\ V_{g}^{*} \xi_{g} & h=1 .\end{cases}
$$

Then $U_{g}=U_{g}^{-1}, g \in G$.

For $g \in G$, define $\Phi_{g}^{0}: B_{0} \rightarrow B_{0}$ by

$$
\left(\Phi_{g}^{0} f\right)_{h}=U_{h} U_{h g}^{-1} f_{h g} U_{h g} U_{h}^{-1} \text { for } f \in B_{0}, h \in G .
$$


Then $\Phi^{0}$ is a homomorphism from $G$ into the *-automorphisms of $B_{0}$. We can embed $A$ as a $C^{*}$-subalgebra of $B_{0}$ as follows: Define $i: A \rightarrow B_{0}$ by

$$
(i a)_{h}=\bigoplus_{g \in G} \pi_{g}(a)=\pi(a) \text { say, } \quad a \in A, h \in G .
$$

The identity of $A$ is then the identity of $B_{0}$. Now define $N^{0}: B_{0} \rightarrow B(\mathscr{H})$ by $N^{0} f=$ $W^{*} f_{1} W$, where $W$ is the isometry from $\mathscr{H}$ into $R$ defined by

$$
(W \xi)_{h}= \begin{cases}0 & h \neq 1 \\ \xi & h=1 .\end{cases}
$$

Then it can be checked that $N^{0} \Phi_{g}^{0}(i a)=w_{g}(a) \quad \forall g \in G, a \in A$.

We now indicate how to prove that if $g_{i} \in G, a_{i} \in A, i=1,2, \ldots, n$, then

$N^{0} \Phi_{g_{n}}^{0}\left(a_{n}\right) \ldots \Phi_{g_{1}}^{0}\left(a_{1}\right) \in A$.

In order to do this, we first show by induction on $n$, that if $\xi \in \mathscr{H}$ is kept fixed

$$
U_{g_{n}} \pi\left(a_{n}\right) U_{g_{n}} U_{g_{n-1}} \pi\left(a_{n-1}\right) \ldots U_{g_{2}} U_{g_{1}} \pi\left(a_{1}\right) U_{g_{1}} W \xi=\eta_{h}^{n}
$$

where for each $h$ in $G, \eta_{h}^{n}$ is a finite sum of terms each of the form

$$
\begin{aligned}
& \pi_{h}(x) V_{h} y \xi \quad x, y \in A . \\
& \text { i.e. } \eta_{h}^{n}=\sum_{i=1}^{p} \pi_{h}\left(x_{i}\right) V_{h} y_{i} \xi
\end{aligned}
$$

where $\left(p, x_{1}, \ldots, x_{p}, y_{1}, \ldots, y_{p}\right)$ depend on $n, h$ and are independent of $\xi \in \mathscr{H}$. It follows that

$$
N^{0} \Phi_{g_{n}}^{0}\left(a_{n}\right) \ldots \Phi_{g_{1}}^{0}\left(a_{1}\right) \xi=\eta_{1}^{n} .
$$

But by the above induction, $\eta_{1}^{n}=x \xi$ where $x \in A$.

Hence

$$
N^{0} \Phi_{g_{n}}^{0}\left(a_{n}\right) \ldots \Phi_{g_{1}}^{0}\left(a_{1}\right) \in A \text {. }
$$

Let $B$ be the $C^{*}$-algebra generated by $\left\{\Phi_{g}^{0}(a): g \in G, a \in A\right\}$. Then $\Phi_{g}^{0}$ leaves $B$ invariant and if $\Phi_{g}=\left.\Phi_{g}^{0}\right|_{B}, g \rightarrow \Phi_{g}$ is a homomorphism from $G$ into the *automorphisms of $B$. Let $N=\left.N^{0}\right|_{B}$. Then $N$ is a projection of norm one of $B$ onto $A$, hence a conditional expectation. The remainder of the proof is clear.

Note that we can easily modify the proof of the above theorem to obtain similar results for $W^{*}$-algebras and $\Sigma^{*}$-algebras with families of $W^{*}$ and $\Sigma^{*}$ continuous $C P$ maps respectively.

We can obtain a number of corollaries from Theorem 1 . The first of which corresponds to Nagy's theorem [12] for unitary dilations of contraction semigroups on a Hilbert space, and E. Stroescu's Banach space analogue [11, Corollary 1].

Corollary 1. Let $\left\{w_{t}: t \in \mathbb{R}^{+}\right\}$be a family of $C P$ maps from a $C^{*}$-algebra $A$ with identity into itself, such that $w_{0}=1$ and $w_{t} 1=1, t \geqq 0$. Then there exists a $C^{*}$-algebra $B$ containing $A$, a one parameter group of *-automorphisms $\Phi_{t}$ of $B$, and a normal conditional expectation $N$ of $B$ onto A satisfying: 
i) $N \Phi_{t}(a)=w_{|t|}(a) \forall a \in A, t \in \mathbb{R}$.

ii) $B$ is the $C^{*}$-algebra generated by $\left\{\Phi_{t}(a): t \in \mathbb{R}, a \in A\right\}$.

iii) The identity of $A$ is also an identity for $B$.

The Hilbert space and Banach space analogues of the following can be seen in [12] and [11] respectively.

Corollary 2. Let $w$ be a CP map from a $C^{*}$-algebra $A$ with identity into itself satisfying $w 1=1$. Then there exists a $C^{*}$-algebra $B$ containing $A$, a normal conditional expectation $N$ from $B$ onto $A$, and $a *$-automorphism $\Phi$ of $B$ onto $A$ satisfying

i) $N \Phi^{n}(a)=w^{|n|}(a) \quad \forall a \in A, n \in \mathbb{Z}$.

ii) $B$ is the $C^{*}$-algebra generated by $\left\{\Phi^{n}(a): a \in A, n \in \mathbb{Z}\right\}$.

iii) The identity of $A$ is also an identity for $B$.

The next corollary corresponds to Ando's result for unitary dilations of a pair of commuting contractions on a Hilbert space [1]. As in the Banach space case, [11], we can take a finite number of not necessarily commuting operators.

Corollary 3. Let $w_{1}, \ldots, w_{p}$ be a finite system of (not necessarily commuting) $C P$ maps of a $C^{*}$-algebra $A$ with identity into itself satisfying $w_{i} 1=1$ for $i=1, \ldots, p$. Then there exists a $C^{*}$-algebra $B$ containing $A$, a normal conditional expectation $N$ of $B$ onto $A$, a finite system of commuting *-automorphisms $\Phi_{1}, \ldots, \Phi_{p}$ of $B$ satisfying

i) $N \Phi_{1}^{n_{1}} \ldots \ldots \circ \Phi_{p}^{n_{p}}(a)=w_{1}^{\left|n_{1}\right|} \ldots \ldots w_{p}^{\left|n_{p}\right|}(a)$ for all $a$ in $A n_{1}, \ldots, n_{p}$ in $\mathbb{Z}$.

ii) $B$ is the $C^{*}$-algebra generated by

$\left\{\Phi_{1}^{n_{1}} \ldots \circ \Phi_{p}^{n_{p}}(a): a \in A, n_{1}, \ldots, n_{p} \in \mathbb{Z}\right\}$.

iii) The identity of $A$ is also an identity for $B$.

Again there are $W^{*}$ and $\Sigma^{*}$-analogues of Corollaries 1-3.

\section{Some Schwarz Type Inequalities for $\boldsymbol{n}$-Positive Linear Mappings on *-Algebras}

Suppose $\mathscr{H}$ is a Hilbert space and $C \in B(\mathscr{H})^{+}$. Then for all $y$ in $B(\mathscr{H}), \varepsilon>0$, $y^{*}(C+\varepsilon)^{-1} y \in B(\mathscr{H})$ and increases monotonically as $\varepsilon \downarrow 0$. Hence

$$
\text { st } \lim _{\varepsilon \downarrow 0} y^{*}(C+\varepsilon)^{-1} y
$$

exists if $y^{*}(C+\varepsilon)^{-1} y$ is bounded above for all $\varepsilon>0$. We write this limit as $y^{*} C^{-1} y$ [7]. If $V$ is a vector subspace of $B(\mathscr{H})$ and $y^{*} C^{-1} y$ exists for all $y$ in $V$, then st $\lim _{\varepsilon \downarrow 0} y^{*}(C+\varepsilon)^{-1} x$ exists for all $x, y$ in $V$, and we write this as $y^{*} C^{-1} x$.

The following theorem is already known in the following special cases. Suppose $A$ is a $C^{*}$-algebra, and $w: A \rightarrow B(\mathscr{H})$ is linear. Then

a) If $w$ is $C P, w\left(x^{*} x\right) \geqq w\left(x^{*} y\right) w\left(y^{*} y\right)^{-1} w\left(y^{*} x\right) \forall x, y \in A$ [7].

b) If $A$ has an identity and $w$ is 2-positive with $w(1) w(x)=w(x) \forall x \in A$, then $w\left(x^{*} x\right) \geqq w\left(x^{*}\right) w(x) \forall x \in A[2]$.

Theorem 2. Let $A$ be $a{ }^{*}$-algebra, $\mathscr{H}$ a Hilbert space and $w: A \rightarrow B(\mathscr{H})$ an $n$ positive *linear map, where $n \geqq 2$. Then if $y, x_{i} \in A, i=1,2, \ldots, n-1$, we have

$$
\left[w\left(x_{i}^{*} x_{j}\right)\right] \geqq\left[w\left(x_{i}^{*} y\right) w\left(y^{*} y\right)^{-1} w\left(y^{*} x_{j}\right)\right] \text { in } B(\mathscr{H})_{n-1}^{-} .
$$


In particular

$\left\|w\left(y^{*} y\right)\right\|\left[w\left(x_{i}^{*} x_{j}\right)\right] \geqq\left[w\left(x_{i}^{*} y\right) w\left(y^{*} x_{j}\right)\right]$.

Proof. Put $x_{0}=y$. Then if $\xi_{0}, \xi_{1}, \ldots, \xi_{n-1} \in \mathscr{H}$,

$\sum_{i, j=0}^{n-1}\left\langle w\left(x_{i}^{*} x_{j}\right) \xi_{j}, \xi_{i}\right\rangle \geqq 0 \quad$ since $w$ is $n$-positive;

i.e.

$$
\begin{aligned}
& \sum_{i, j=1}^{n-1}\left\langle w\left(x_{i}^{*} x_{j}\right) \xi_{j}, \xi_{i}\right\rangle+\sum_{i=1}^{n-1}\left\langle w\left(x_{i}^{*} y\right) \xi_{0}, \xi_{i}\right\rangle \\
+ & \sum_{i=1}^{n-1}\left\langle w\left(y^{*} x_{i}\right) \xi_{i}, \xi_{0}\right\rangle+\left\langle w\left(y^{*} y\right) \xi_{0}, \xi_{0}\right\rangle \geqq 0 .
\end{aligned}
$$

We now fix $\xi_{1}, \ldots, \xi_{n-1}$ and put $\xi_{0}=-\left[w\left(y^{*} y\right)+\varepsilon\right]^{-1} \sum_{i=1}^{n-1} w\left(y^{*} x_{i}\right) \xi_{i}$. Then

$$
\begin{gathered}
\sum_{i, j=1}^{n-1}\left\langle w\left(x_{i}^{*} x_{j}\right) \xi_{j}, \xi_{i}\right\rangle-2 \sum_{i, j=1}^{n-1}\left\langle x\left(x_{i}^{*} y\right)\left[w\left(y^{*} y\right)+\varepsilon\right]^{-1} w\left(y^{*} x_{j}\right) \xi_{j}, \xi_{i}\right\rangle \\
+\sum_{i, j=1}^{n-1}\left\langle w\left(x_{i}^{*} y\right)\left[w\left(y^{*} y\right)+\varepsilon\right]^{-1} w\left(y^{*} y\right)\left[w\left(y^{*} y\right)+\varepsilon\right]^{-1} w\left(y^{*} x_{j}\right) \xi_{j}, \xi_{i}\right\rangle \geqq 0 .
\end{gathered}
$$

But $2\left[w\left(y^{*} y\right)+\varepsilon\right]^{-1}-\left[w\left(y^{*} y\right)+\varepsilon\right]^{-2} w\left(y^{*} y\right)=\left[w\left(y^{*} y\right)+\varepsilon\right]^{-1}+\varepsilon\left[w\left(y^{*} y\right)+\varepsilon\right]^{-2}$.

Hence

$$
\begin{aligned}
& \sum_{i, j=1}^{n-1}\left\langle w\left(x_{i}^{*} x_{j}\right) \xi_{j}, \xi_{i}\right\rangle \\
\geqq & \sum_{i, j=1}^{n-1}\left\langle w\left(x_{i}^{*} y\right)\left\{\left[w\left(y^{*} y\right)+\varepsilon\right]^{-1}+\varepsilon\left[w\left(y^{*} y\right)+\varepsilon\right]^{-2}\right\} w\left(y^{*} x_{j}\right) \xi_{j}, \xi_{i}\right\rangle \\
\geqq & \sum_{i, j=1}^{n-1}\left\langle w\left(x_{i}^{*} y\right)\left[w\left(y^{*} y\right)+\varepsilon\right]^{-1} w\left(y^{*} x_{j}\right) \xi_{j}, \xi_{i}\right\rangle .
\end{aligned}
$$

The remainder of the proof is clear, and is omitted.

Corollary 4. $A$ is a Banach *-algebra with approximate identity and $w: A \rightarrow B(\mathscr{H})$ is an n-positive bounded linear map. Then for all $x_{i} \in A, i=1,2, \ldots, n-1$

$\|w\|\left[w\left(x_{i}^{*} x_{j}\right)\right] \geqq\left[w\left(x_{i}^{*}\right) w\left(x_{j}\right)\right]$.

Remark 1 . We note that Theorem 2 and Corollary 4 can be used to characterise complete positivity.

Corollary. If $A$ is $a^{*}$-algebra, $\mathscr{H}$ a Hilbert space, and $w: A \rightarrow B(\mathscr{H})$ is $a *$-linear map. Then $w$ is completely positive iff for all $n \geqq 1$,

$y, x_{i} \in A, \quad i=1, \ldots, n$ that $\left[w\left(x_{i}^{*} x_{j}\right)\right] \geqq\left[w\left(x_{i}^{*} y\right) w\left(y^{*} y\right)^{-1} w\left(y^{*} x_{j}\right)\right]$.

Corollary. If $A$ is a Banach *algebra with approximate identity, $\mathscr{H}$ a Hilbert space and $w: A \rightarrow B(\mathscr{H})$ a bounded linear map. Then $w$ is completely positive iff for 
all $n \geqq 1, x_{i} \in A_{i}, i=1,2, \ldots, n$ that

$$
\|w\|\left[w\left(x_{i}^{*} x_{j}\right)\right] \geqq\left[w\left(x_{i}\right) * w\left(x_{j}\right)\right] .
$$

Remark 2. It follows from Corollary 4 that if $A, B$ are $C^{*}$-algebras and $w: A \rightarrow B$ is an invertible linear map such that $w$ and $w^{-1}$ are 2-positive, with $\|w\|=1=$ $\left\|w^{-1}\right\|$, then $w$ is a ${ }^{*}$-isomorphism. We will show in the next section that is possible to have a $C^{*}$-algebra $A$ with identity, and $w: A \rightarrow A$ an invertible linear map such that $w$ is completely positive, but $w^{-1}$ is not 2-positive.

\section{Strongly Continuous Semigroups and Groups of Positive Maps on $C^{*}$-Algebras}

Let $A$ be a $C^{*}$-algebra with identity and $\theta$ a completely positive linear map of $A$ into itself. Following Lindblad [9], we define

$$
L_{\theta}(x)=\theta(x)-\frac{1}{2}\{\theta(1), x\} \quad x \in A .
$$

Then $\left\{e^{t L_{\theta}}: t \geqq 0\right\}$ is a norm continuous one-parameter semigroup of completely positive maps on $A$, which preserve the identity, [9].

From the identity

$$
L_{\theta}\left(x^{*} x\right)-L_{\theta}\left(x^{*}\right) \cdot x-x^{*} \cdot L_{\theta}(x)=\left[\pi_{\theta}(x) V_{\theta}-V_{\theta} x\right]^{*}\left[\pi_{\theta}(x) V_{\theta}-V_{\theta} x\right], \quad x \in A
$$

we deduce:

Proposition 1. The following conditions are equivalent:

i) $L_{\theta}$ is a derivation.

ii) $L_{\theta}=0$.

iii) $\theta(x)=\theta(1) x, \forall x \in A$ (and $\theta(1)$ lies in the centre of $A$ ).

iv) $\pi_{\theta}(x) V_{\theta}=V_{\theta} x, \forall x \in A$.

We now introduce a hamiltonian term. Let $\left\{e^{t \delta}: t \geqq 0\right\}$ be a strongly continuous one-parameter semigroup of *-homomorphisms of $A$. Then using the Lie-Trotter formula as in [5] and [9], we see that $\left\{\exp t\left(\delta+L_{\theta}\right): t \geqq 0\right\}$ is a strongly continuous one-parameter semigroup of identity preserving completely positive maps on $A$. Proposition 1 yields the following:

Theorem 3. Define $L=L_{\theta}+\delta$. Then the following conditions are equivalent:

i) $\exp t L$ is a group of *-automorphisms.

ii) $\exp t L$ is a group of CP maps.

iii) $\exp t L$ is a group of 2-positive maps.

iv) $L=\delta$.

v) $\theta(x)=\theta(1) x, \forall x \in A$.

vi) $\pi_{\theta}(x) V_{\theta}=V_{\theta} x, \forall x \in A$.

Now let $\mathscr{H}$ be a Hilbert space, and consider $A=B(\mathscr{H})$. Suppose $V \in B(\mathscr{H})$, $V \notin \mathbb{C} 1$. Define $\theta(x)=V^{*} x V \quad x \in A$. It is then seen that $\exp t L_{\theta}$ is never 2-positive if $t<0$, but is always $C P$ if $t \geqq 0$.

It is also possible to have a group of $C P$ maps on a $C^{*}$-algebra, which is not a group of *-automorphisms. Let $A$ be a $C^{*}$-algebra, and $K$ a non zero self adjoint element of $A$. Then $\alpha_{t}(x)=e^{K t} x e^{K t}$ for $t \in \mathbb{R}$, defines a norm continuous oneparameter group of completely positive maps, but $\alpha_{t}$ is a $*$-automorphism iff 
$t=0$. We shall see in Theorem 4 that this is essentially how all norm continuous one parameter groups of $C P$ maps (and indeed 2-positive maps) on $C^{*}$-algebras arise. For this we need the following lemma, which generalises a part of [9, Cor. 2].

Lemma 1. Let $A$ be a $C^{*}$-algebra with identity and $\exp t L, t \geqq 0$, a strongly continuous one-parameter semigroup of 2-positive maps on $A$, such that $D(L)$ is a subalgebra of $A$, and $1 \in D(L)$. Then for all $x$ in $D(L)$ :

$$
L\left(x^{*} x\right)+x^{*} L(1) x-x^{*} L(x)-L\left(x^{*}\right) x \geqq 0 .
$$

Proof. This follows from Theorem 2 if we first suitably group the terms in

$$
\frac{e^{t L}\left(x^{*} x\right)-x^{*} x}{t} \geqq \frac{1}{t}\left\{e^{t L}\left(x^{*}\right)\left[\varepsilon+e^{t L}(1)\right]^{-1} e^{t L}(x)-x^{*} x\right\}
$$

take a Taylor expansion $e^{t L}(1)=1+L(1) t+o(t)$, and then let $t, \varepsilon \downarrow 0$ with $\varepsilon / t \rightarrow 0$.

Theorem 4. Let $A$ be a $C^{*}$-algebra with identity, and $\{\exp t L: t \in \mathbb{R}\}$ a strongly continuous one parameter group of 2-positive maps on $A$ such that $D(L)$ is a subalgebra of $A$, and $1 \in D(L)$. Then if we define

$$
D(\delta)=D(L), \quad \text { and } \quad \delta x=L x-\frac{1}{2}\{x, L(1)\}, \quad x \in D(\delta),
$$

$\delta$ is a $*$-derivation, and $\exp t L$ is a group of $C P$ maps on $A$.

Moreover, the following conditions are equivalent:

i) $L$ is $a *$-derivation.

ii) $L(1)=0$.

iii) $e^{L t}$ is a group of *automorphisms.

iv) $e^{L t} 1=1 \quad \forall t \in \mathbb{R}$.

v) $\left\|e^{L t} 1\right\|=1 \quad \forall t \in \mathbb{R}$.

We also have the following partial converse to Lemma 1.

Theorem 5. Let $A$ be a $C^{*}$-algebra, and $e^{L t}$ a strongly continuous one parameter semigroup of positive maps on $A$ such that

i) $D(L)$ is a subalgebra of $A$.

ii) $L\left(x^{*} x\right)-x^{*} L(x)-L\left(x^{*}\right) x \geqq 0 \quad \forall x \in D(L)$. Then $e^{L t}\left(x^{*} x\right) \geqq e^{L t}\left(x^{*}\right) e^{L t}(x) \forall x \in A, t \geqq 0$, and hence $e^{L t}$ is a contraction semigroup.

Proof. Let $f \in D(L)$ and define $x(t)=e^{L t} f^{*} f-e^{L t} f^{*} e^{L t} f, t \geqq 0$.

Then $x$ is continuously differentiable and for $t \geqq 0$

$$
x^{\prime}(t)=L e^{L t} f^{*} f-\left(L e^{L t} f^{*}\right) e^{L t} f-e^{L t} f^{*}\left(L e^{L t} f\right) .
$$

Moreover if $h \in D(L)$, then $s \rightarrow e^{L(t-s)} h$ is continuously differentiable in $0 \leqq s \leqq t$ and

$$
\frac{\partial}{\partial s} e^{(t-s) L} h=-e^{L(t-s)} L h .
$$


Hence

$$
\begin{aligned}
& x(t)-e^{L t} x(0) \\
& =\int_{0}^{t} \frac{\partial}{\partial \sigma} e^{(t-\sigma) L} x(\sigma) d \sigma \\
& =-\int_{0}^{t} e^{(t-\sigma) L} L x(\sigma) d \sigma+\int_{0}^{t} e^{(t-\sigma) L} \frac{d}{d \sigma} x(\sigma) d \sigma \\
& =\int_{0}^{t} e^{(t-\sigma) L}\left[L\left(e^{L \sigma} f^{*} e^{L \sigma} f\right)-\left(L e^{L \sigma} f^{*}\right) e^{L \sigma} f-e^{L \sigma} f^{*}\left(L e^{L \sigma} f\right)\right] d \sigma .
\end{aligned}
$$

But by hypothesis, $L\left(e^{L \sigma} f^{*} e^{L \sigma} f\right) \geqq L\left(e^{L \sigma} f^{*}\right) e^{L \sigma} f+e^{L \sigma} f^{*}\left(L e^{L \sigma} f\right)$ for all $f \in D(L)$, $\sigma \geqq 0$. Moreover $e^{L(t-\sigma)}$ is positive in $0 \leqq \sigma \leqq t$. Hence $x(t) \geqq e^{t L} x(0)=0, \forall t \geqq 0$. By density of $D(L)$ in $A$ we deduce:

$$
e^{L t}\left(x^{*} x\right) \geqq e^{L t} x^{*} e^{L t} x, \forall x \in A, t \geqq 0 .
$$

Remark. The proof of Theorem 5 can be adapted to prove the following:

Proposition. Let $L$ be the infinitesimal generator of a strongly continuous oneparameter semigroup on a Banach algebra. Then $L$ is a derivation if and only if $e^{L t}$ is a semigroup of homomorphisms.

\section{References}

1. Ando, T.: Acta Sci. Math. (Szeged) 24, 88-90 (1963)

2. Araki, H.: Publ. Res. Inst. Math. Sci., Kyoto Univ. 8, 439-469 (1972-73)

3. Arveson, W. B.: Acta Math. 123, 141-224 (1969)

4. Davies, E. B. : Z. Wahrscheinlichkeitstheorie verw. Geb. 23, 261-273 (1972)

5. Davies, E. B.: Commun. math. Phys. 39, 91-110 (1974)

6. Evans, D.E.: Thesis, Oxford (1975)

7. Lieb, E. H., Ruskai, M. B.: Advan. Math. 12, 269-273 (1974).

8. Lindblad, G.: Commun. math. Phys. 40, 147-151 (1975)

9. Lindblad, G.: On the generators of quantum dynamical semigroups, Preprint, Royal Institute of Technology, Stockholm (1975)

10. Stinespring, W.F.: Proc. Amer. Math. Soc. 6, 211-216 (1955)

11. Stroescu, E. : Pacific J. Math. 47, 257-267 (1973)

12. Sz.-Nagy, B., Foias, C.: Harmonic analysis of operators on hilbert space. Amsterdam: North Holland Publ. Co. 1970

Communicated by H. Araki

Received November 15, 1975; in revised form January 19, 1976

Note Added in Proof. A Schwarz-type inequality for 2-positive maps has also been proved by M. D. Choi, and appears in Illinois J. Math. 18, 565-574 (1974) 